\title{
PERFORMANCE ANALYSIS FOR PATH ATTENUATION ESTIMATION OF MICROWAVE SIGNALS DUE TO RAINFALL AND BEYOND
}

\author{
Boming Song, Defeng (David) Huang, Xi Shen, and Roberto Togneri
}

Department of Electrical, Electronic, and Computer Engineering, The University of Western Australia, Perth, Australia

\begin{abstract}
The attenuation of microwave signals can be used for meteorological observations. For example, the received signal level (RSL) of backhaul links of cellular systems, which usually has a quantization error of $0.1 \mathrm{~dB}$ or more for commercial systems, has been used to measure rainfall. In this work, through the mean square error (MSE) analysis of an ideal RSL estimator, it is found that the estimation error can be lower than $0.01 \mathrm{~dB}$ for high signal-to-noise ratio (SNR), thereby making it feasible to measure other meteorological variables such as water vapor and clouds. However, the RSL-based estimator has poor performance in low SNR. To improve the performance, we propose a new path attenuation measurement method based on SNR estimation. Although the performance of the SNR-based estimator is better than the RSL based one for low SNR, it becomes worse in high SNR when the path attenuation is small. To solve the problem, another method is proposed based on estimating the signal power (SP) only. Both MSE analysis and simulation results show that the SP-based method is superior to both RSL and SNR based estimators for most scenarios.
\end{abstract}

Index Terms - Microwave attenuation, RSL, SNR estimation, signal power estimation, MSE

\section{INTRODUCTION}

Rainfall observation by using microwave links, including backhaul links of the commercial wireless communication networks (CWCNs) [1] and the communication links of satellites [2], is a complement to traditional methods of rainfall measurement (e.g., rain gauge, weather radar, and meteorological satellites). The microwave-based method can also be used to measure other meteorological variables such as clouds [3] and dust storm [4]. The well-known K-R relationship [5] is fundamental to measure rainfall. The relationships between microwave attenuation and clouds [3] or dust storm [4] have also been established. However, the attenuation caused by those weather phenomena is less than that caused by precipitation. For example, the water content of the water vapor in the cloud ranges from $0.4 \mathrm{~g} / \mathrm{m}^{3}$ to $1 \mathrm{~g} / \mathrm{m}^{3}$ [3], and in some nonprecipitating clouds, it is even lower than $0.1 \mathrm{~g} / \mathrm{m}^{3}$ [6]. The attenuation caused by such low water content is between $0.01 \mathrm{~dB} / \mathrm{km}$ and $0.1 \mathrm{~dB} / \mathrm{km}$ for a $35 \mathrm{GHz}$ signal [7], which is much lower than that caused by rainfall. For measuring such low attenuation, higher resolution is required.

Many works have investigated the rainfall observation by using the RSL of backhaul links of CWCNs [1], [8] - [10]. However, the RSL data are stored digitally with a typical quantization error of 1 $\mathrm{dB}$ or $0.1 \mathrm{~dB}$ [11], and there is no work dedicated to analyzing the performance of the RSL-based attenuation estimator that can be achieved if the quantization error is not a limitation. Besides the
RSL, a method of rainfall measurement using the carrier-to-noise ratio (CNR) estimation of satellite communication links was proposed in [12]. The CNR is essentially the signal-to-noise ratio (SNR) of a modulated signal. An SNR-based method was proposed by Shen et al. [2] for rain field reconstruction using microwave signals from low earth orbit satellites. However, the performance of the SNR estimator on the rain attenuation estimation has not been analyzed.

In this paper, firstly, we analyze both the RSL-based and the SNR-based path attenuation estimation methods. With the use of an ideal model of the RSL estimator, the performance limit of the RSLbased method is analyzed. For the SNR-based method, we derive the theoretical limit using the maximum likelihood (ML) estimator with the transmitted data known at the receiver [13] - [14]. Secondly, we propose a new method of path attenuation measurement based on signal power (SP) estimation. The theoretical limit of its performance is also given. For all the three methods, the path attenuation is obtained by measurements in clear and unclear (i.e., 'unclear' ('clear') means affected (not affected) by rainfall, fog, clouds or other weather events) weather situation, separately. Aspects of the impact on the performance of the path attenuation estimation are discussed in this work, which includes the observed signal window length, the value of the path attenuation, and the SNR operating points at the receiver. According to the results, the SPbased method shows the best performance for most cases. These improvements may make it feasible to use the microwave links to measure other meteorological variables other than rain in the future.

\section{SIGNAL MODEL}

Consider an MPSK signal for a microwave link modeled by an AWGN channel and the equivalent baseband signal $\tilde{s}$ is given by:

$$
\tilde{s}=a_{I}+j a_{Q}
$$

where $a_{I}, a_{Q}$ are the transmitted data of in-phase and quadrature components. The transmitted RF signal is:

$$
s(t)=\sqrt{\frac{2}{T}} \operatorname{Re}\left[\tilde{s} e^{j 2 \pi f_{c} t}\right], \quad 0 \leq t<T
$$

where $f_{c}$ is the carrier frequency, and $T$ is the symbol duration. At the receiver, the received signal is given by:

$$
r(t)=\sqrt{P_{s}} s(t)+\omega(t)
$$

where $P_{S}$ denotes the signal power, and $\omega(t)$ is bandlimited white Gaussian process with zero-mean and variance of $N_{0} B\left(N_{0}\right.$ is the power spectral density of the AWGN, and $B$ is the bandwidth of the MPSK signal). $\omega(t)$ can also be expressed as [15]:

$$
\omega(t)=\omega_{1} \phi_{1}(t)+\omega_{2} \phi_{2}(t)+\omega_{3} \phi_{3}(t)
$$

where $\phi_{1}(t)$ and $\phi_{2}(t)$ are two normalized basis functions defined by: 


$$
\begin{array}{cc}
\phi_{1}(t)=\sqrt{\frac{2}{T}} \cos 2 \pi f_{c} t, & 0 \leq t<T \\
\phi_{2}(t)=-\sqrt{\frac{2}{T}} \sin 2 \pi f_{c} t, & 0 \leq t<T
\end{array}
$$

and $\phi_{3}(t)$ is a normalized basis function orthogonal to $\phi_{1}(t)$ and $\phi_{2}(t)$. The coefficients $\omega_{i}, i=1,2,3$ are Gaussian variables with zero mean and variance of $\sigma^{2}=N_{0} B$.

Then, Eq. (3) can be represented by the orthonormal basis functions $\phi_{i}(t), i=1,2,3$ :

$$
\begin{aligned}
r(t)= & \left(\sqrt{P_{s}} a_{I}+\omega_{1}\right) \phi_{1}(t) \\
& +\left(\sqrt{P_{s}} a_{Q}+\omega_{2}\right) \phi_{2}(t)+\omega_{3} \phi_{3}(t)
\end{aligned}
$$

\section{PATH ATTENUATION MEASUREMENT}

\subsection{RSL-based attenuation estimator}

Using Eq. (6) and the property of $\int_{-\infty}^{\infty} \phi_{i}(t) \phi_{j}(t) d t=0$ for $i \neq j$, the received power for one symbol at the RF or intermediate frequency (IF) is given by:

$$
\begin{aligned}
\hat{P}_{\text {sym }} & =\int_{0}^{T}|r(t)|^{2} d t \\
& =\left(\sqrt{P_{s}} a_{I}+\omega_{1}\right)^{2}+\left(\sqrt{P_{s}} a_{Q}+\omega_{2}\right)^{2}+\omega_{3}^{2}
\end{aligned}
$$

The time window for measuring an instantaneous RSL value often ranges from tens to hundreds of microseconds. Assume that a signal sequence consisting of $L$ symbols is received to measure the RSL within the time window. The average received power of the $L$ symbols is given by:

$$
\hat{P}=\frac{1}{L} \sum_{n=1}^{L}\left[\left(\sqrt{P_{s}} a_{I, n}+\omega_{1, n}\right)^{2}+\left(\sqrt{P_{S}} a_{Q, n}+\omega_{2, n}\right)^{2}+\omega_{3, n}^{2}\right]
$$

where the subscript $n$ is the index corresponding to the $n$-th transmitted symbol. The output instantaneous RSL value in decibels can be expressed as:

$$
\widehat{R S L}=10 \cdot \log _{10}(\widehat{P})
$$

In this paper, we assume that the path attenuation is obtained by measuring the received signals twice, one in the clear weather situation and another in the unclear weather situation. Note that such an approach has often been used in rain measurement using CWCNs [16]-[17]. It is also assumed that the transmitted powers and the noise powers are the same for the transmissions during the two weather situation.

Let $\widehat{R S} L_{c}$ denote the estimated RSL in the clear weather situation and $\widehat{R S} L_{u}$ denote that in the unclear weather situation. The path attenuation $\hat{\alpha}$ can be retrieved by:

$$
\hat{\alpha}=\widehat{R S} L_{c}-\widehat{R S} L_{u}
$$

\subsection{SNR-based attenuation estimator}

The third term in Eq. (6) can be removed by using a matched filter at the receiver, and the output of the matched filter $\widetilde{\boldsymbol{y}}=\left[\tilde{y}_{1}, \tilde{y}_{2}\right]^{T}$ is given by [15]:

$$
\begin{aligned}
\tilde{\boldsymbol{y}} & =\sqrt{P_{S}} \tilde{\boldsymbol{s}}+\widetilde{\boldsymbol{\omega}} \\
\text { where } \tilde{\boldsymbol{S}}=\left[a_{I}, a_{Q}\right]^{T} \text { and } \widetilde{\boldsymbol{\omega}} & =\left[\omega_{1}, \omega_{2}\right]^{T}
\end{aligned}
$$

Assume that the transmitted data is known at the receiver and a total of $L$ symbols are received, the log-likelihood function: $\ln F\left(\widetilde{\boldsymbol{y}}_{n}, n=1,2, \ldots, L \mid P_{s}, \sigma^{2}\right)=-L \ln \left(2 \pi \sigma^{2}\right)-$

$$
\frac{1}{2 \sigma^{2}}\left[\sum_{n=1}^{L}\left(\tilde{y}_{1, n}-\sqrt{P_{S}} a_{I, n}\right)^{2}+\sum_{n=1}^{L}\left(\tilde{y}_{2, n}-\sqrt{P_{S}} a_{Q, n}\right)^{2}\right]
$$

where $\tilde{\boldsymbol{y}}_{n}=\left[\tilde{y}_{1, n}, \tilde{y}_{2, n}\right]^{T}$ is the output of the matched filter corresponding to the $\mathrm{n}$-th symbol $\left[a_{I, n}, a_{Q, n}\right]^{T}$.

By differentiating Eq. (12) with respect to $P_{S}$ and $\sigma^{2}$, and setting the derivative equal to zero, separately, we obtain $\hat{P}_{S}$ and $\hat{\sigma}^{2}$ :

$$
\begin{gathered}
\hat{P}_{S}=\left[\frac{\sum_{n=1}^{L}\left(\tilde{y}_{1, n} a_{I, n}+\tilde{y}_{2, n} a_{Q, n}\right)}{\sum_{n=1}^{L}\left(a_{I, n}^{2}+a_{Q, n}^{2}\right)}\right]^{2} \\
\hat{\sigma}^{2}=\frac{\sum_{n=1}^{L}\left(\tilde{y}_{1, n}-\sqrt{\widehat{P}_{S}} a_{I, n}\right)^{2}+\sum_{n=1}^{L}\left(\tilde{y}_{2, n}-\sqrt{\widehat{P}_{S}} a_{Q, n}\right)^{2}}{2 L}
\end{gathered}
$$

The estimated SNR in decibel is then given by:

$$
\widehat{S N R}=10 \log _{10}\left(\frac{\hat{P}_{S}}{\hat{\sigma}^{2}}\right)
$$

Let $\widehat{S N R}_{c}$ denote the estimated SNR in clear weather situation and $\widehat{S N R}_{u}$ denote that in the unclear weather situation. Then, the path attenuation can be estimated by:

$$
\hat{\alpha}=\widehat{S N R}_{c}-\widehat{S N R}_{u}
$$

\subsection{SP-based attenuation estimator}

For the SNR-based estimator in Section 3.2, the signal power and noise power are estimated separately. As the noise is assumed to be unaffected by the path attenuation, it is natural to estimate the rain attenuation using only the estimated signal power given by Eq. (13). Let $\hat{P}_{s, c}$ denote the estimated signal power in clear weather situation and $\hat{P}_{s, u}$ denote that in the unclear situation. Then, the path attenuation can be estimated by:

$$
\hat{\alpha}=10 \log _{10}\left(\frac{\hat{P}_{s, c}}{\hat{P}_{s, u}}\right)
$$

\section{THE BIAS AND MEAN SQUARE ERROR (MSE) OF THE PATH ATTENUATION ESTIMATION}

The bias and MSE are defined as:

$$
\begin{gathered}
\operatorname{bias}(\hat{\alpha})=E[\hat{\alpha}-\alpha] \\
m s e(\hat{\alpha})=E\left[(\hat{\alpha}-\alpha)^{2}\right]
\end{gathered}
$$

where $E[\cdot]$ denotes the expectation, and $\alpha$ is the path attenuation in decibel. Let $\xi$ denotes the attenuation ratio along the path in linear scale, then $\alpha=10 \log _{10} \xi$.

For the same system setup, the CNR (or SNR of modulated signal) for the RSL-based estimator in clear weather situation is defined as $\rho_{1}=\frac{2 P_{s}}{3 \sigma^{2}}$; for the SNR-based estimator and the SP-based estimator, the corresponding SNR is defined as $\rho_{2}=\frac{P_{s}}{\sigma^{2}}$. As the transmitted powers for clear weather and unclear weather are the same, the CNR for the unclear weather situation is given by $\rho_{1} / \xi$ for the RSL-based estimator and $\rho_{2} / \xi$ for the SNR-based estimator and the SP-based estimator. Note that the SNR for the RSL-based path attenuation estimator is lower than the SNR-based and the SPbased estimator because the noise component is reduced by the matched filter for the SNR-based and SP-based estimators.

In the following, to derive the bias and the MSE of each attenuation estimator, the first-order Taylor expansion of the logarithm function is used to approximate $E\left[\log _{10} X\right]$ and $E\left[\left(\log _{10} X\right)^{2}\right]$. The Taylor expansion of $\log _{10} X$ at $E[X]$ is given by:

and therefore we have:

$$
\log _{10} X \approx \log _{10} E[X]+\frac{X-E[X]}{E[X] \ln 10}
$$

$$
E\left[\log _{10} X\right] \approx \log _{10} E[X]
$$


For $E\left[\left(\log _{10} X\right)^{2}\right]$, the Taylor expansions of $\left(\log _{10} X\right)^{2}$ at $E[X]$ is given by:

$$
\left(\log _{10} X\right)^{2} \approx\left(\log _{10} E[X]\right)^{2}+\frac{2 \log _{10} E[X]}{E[X] \ln 10}(X-E[X])
$$

Then, we have:

$$
E\left[\left(\log _{10} X\right)^{2}\right] \approx\left(\log _{10} E[X]\right)^{2}
$$

\subsection{Performance of the RSL-based path attenuation estimator}

Let $\operatorname{bias}_{1}(, ;)$ and mse $_{1}(,, \cdot)$ denote the bias and the MSE of the RSLbased path attenuation estimator, respectively. By substituting Eq. (8) - (10) into Eq. (18), and using the approximation of Eq. (20), the bias $_{1}$ can be approximated as:

$$
\operatorname{bias}_{1}\left(\rho_{1}, \xi\right) \approx 10 \log _{10}\left(\frac{\rho_{1}+1}{\rho_{1}+\xi}\right)
$$

Using the approximation of Eq. (21), the MSE can be approximated as:

$$
m s e_{1}\left(\rho_{1}, \xi\right) \approx\left[10 \log _{10}\left(\frac{\rho_{1}+1}{\rho_{1}+\xi}\right)\right]^{2}
$$

\subsection{Performance of the SNR-based path attenuation estimator}

Let $\operatorname{bias}_{2}(\because,$,$) and$ mse $_{2}(\because,$,$) denote the bias and the MSE of the$ SNR-based path attenuation estimator, respectively. Using the results in [18] regarding the expected value of the ML-based SNR estimation and the approximation of Eq. $(20)$, the $\operatorname{bias}_{2}(\because, ;)$ can be approximated as:

$$
\operatorname{bias}_{2}\left(\rho_{2}, \xi, L\right) \approx 10 \log _{10}\left(\frac{2 L \rho_{2}+1}{2 L \rho_{2}+\xi}\right)
$$

Using Eq. (21), the $\mathrm{mse}_{2}(\cdot, \xi, L)$ is approximated as:

$$
\begin{array}{r}
\operatorname{mse}_{2}\left(\rho_{2}, \xi, L\right) \approx \operatorname{mse}_{S N R}\left(\rho_{2}, L\right)+\operatorname{mse}_{S N R}\left(\frac{\rho_{2}}{\xi}, L\right) \\
-2 \operatorname{bias}_{S N R}\left(\rho_{2}, L\right) \operatorname{bias}_{S N R}\left(\frac{\rho_{2}}{\xi}, L\right) \\
\geq \operatorname{crlb}_{S N R}\left(\rho_{2}, L\right)+\operatorname{crlb}_{S N R}\left(\frac{\rho_{2}}{\xi}, L\right) \\
-2 \operatorname{bias}_{S N R}\left(\rho_{2}, L\right) \operatorname{bias}_{S N R}\left(\frac{\rho_{2}}{\xi}, L\right)
\end{array}
$$

where $\operatorname{mse}_{S N R}(\because$,$) denotes the MSE of the ML-based SNR$ estimation, and $\operatorname{crlb} b_{S N R}(\because ;)$ denotes the well-known Cramer-Rao lower bound (CRLB) [19] of the SNR estimator, which is given by:

$$
\operatorname{crlb}_{S N R}(x, L)=\frac{100}{L(\ln (10))^{2}}\left(\frac{2}{x}+1\right)
$$

The $\operatorname{bias}_{S N R}(\cdot$,$) in Eq. (25)$ denotes the bias of the ML-based SNR estimation, which is given by:

$$
\operatorname{bias}_{S N R}(x, L)=10 \log _{10}\left[\frac{2 L x+1}{(2 L-3) x}\right]
$$

Note that for $L \rightarrow \infty$, the CRLB approaches the mean square error, as the SNR estimator is ML based and asymptotically unbiased. Similarly for the analysis of SP estimator in the following.

\subsection{Performance of the SP-based path attenuation estimator}

Let $\operatorname{bias}_{3}(\because, ;)$ and $m_{\text {se }}(\because, ;)$ denote the bias and the MSE of the SP-based path attenuation estimator, respectively. By using the approximation of Eq. $(20)$, bias $_{3}(\cdot)$ is approximated as:

$$
\operatorname{bias}_{3}\left(\rho_{2}, \xi, L\right) \approx 10 \log _{10}\left(\frac{2 \rho_{2} L+1}{2 \rho_{2} L+\xi}\right)
$$

which is the same as Eq. (24). Using the approximation of Eq. (21), the $\operatorname{mse}_{3}(\because,$,$) is approximated as:$

$$
m s e_{3}\left(\rho_{2}, \xi, L\right) \approx m s e_{S P}\left(\rho_{2}, L\right)+m s e_{S P}\left(\frac{\rho_{2}}{\xi}, L\right)
$$

$$
\begin{array}{r}
-2 \operatorname{bias}_{S P}\left(\rho_{2}, L\right) \operatorname{bias}_{S P}\left(\frac{\rho_{2}}{\xi}, L\right) \\
\approx \operatorname{crlb}_{S P}\left(\rho_{2}, L\right)+\operatorname{crlb}_{S P}\left(\frac{\rho_{2}}{\xi}, L\right) \\
-\operatorname{2bias}_{S P}\left(\rho_{2}, L\right) \operatorname{bias}_{S P}\left(\frac{\rho_{2}}{\xi}, L\right)
\end{array}
$$

where the $\operatorname{mse}_{S P}(\because)$ is the MSE of the signal power estimator, and the $\operatorname{crlb} b_{S P}(\because$,$) is the CRLB of the SP estimator, which is given by:$

$$
c r l b_{S P}(x, L)=\frac{200}{L(\ln 10)^{2}}\left(\frac{1}{x}\right)
$$

$\operatorname{bias}_{S P}(, ;)$ in Eq. $(28)$ denotes the bias of the SP estimator, which is given by:

$$
\operatorname{bias}_{S P}(x, L)=10 \log _{10}\left(1+\frac{1}{2 L x}\right)
$$

\section{NUMERICAL RESULTS}

The QPSK signal is applied to all the results in this section. All the simulation results are obtained by 10,000 trials. To demonstrate the performance of the three path attenuation estimators, simulations have been carried out for $\alpha=0.5 \mathrm{~dB}, 3 \mathrm{~dB}$, and $18 \mathrm{~dB}$, respectively. Note that for a carrier frequency of $35 \mathrm{GHz}, \alpha=0.5 \mathrm{~dB}$ is about the specific rain attenuation per kilometer for light rain, $\alpha=3 \mathrm{~dB}$ is about the specific rain attenuation per kilometer for heavy rain, and $\alpha=18 \mathrm{~dB}$ is about the path attenuation of several kilometers in the case of heavy rain [20]. In the following, for a fair comparison, when the MSEs of the RSL-based, the SNR-based, and the SP-based estimators are plotted on the figure, the SNR (i.e., $\rho_{2}$ ) is applied to the SNR-based and the SP-based estimators, and the corresponding CNR (i.e., $\rho_{1}$ ) is applied to the RSL-based estimator. As mentioned in Section 4, for the same system setup, the SNR is about $1.8 \mathrm{~dB}$ larger than the CNR.

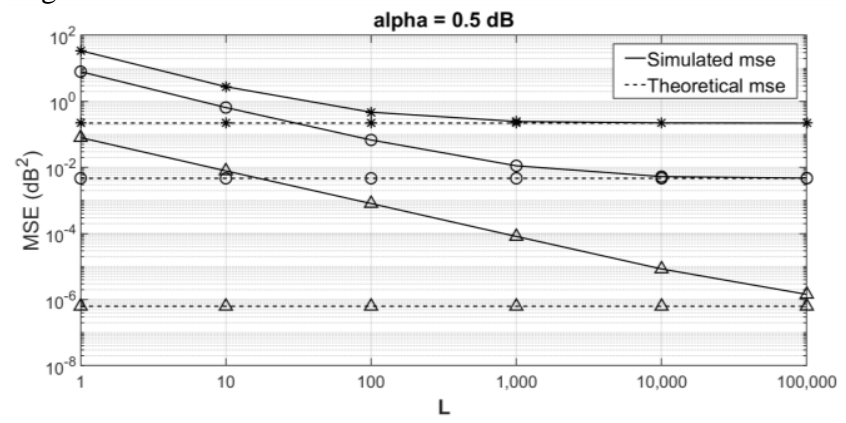

Fig. 1 MSE of the RSL-based attenuation estimator at $\mathrm{CNR}=-11.8$ $\mathrm{dB}(*), 8.2 \mathrm{~dB}(\mathrm{o})$ and $28.2 \mathrm{~dB}(\Delta)$ for $\alpha=0.5 \mathrm{~dB}$.

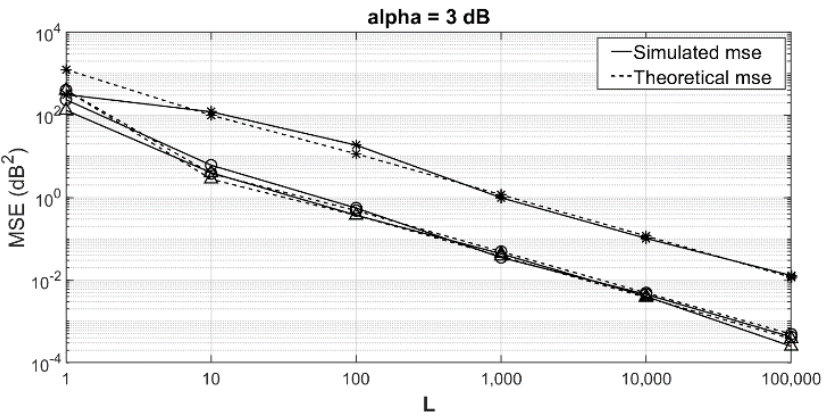

Fig. 2 MSE of the SNR-based attenuation estimator at $\mathrm{SNR}=-10$ $\mathrm{dB}(*), 10 \mathrm{~dB}(\mathrm{o})$ and $30 \mathrm{~dB}(\Delta)$ for $\alpha=3 \mathrm{~dB}$.

To demonstrate the effect of the using the approximations of Eq. (20) and (21), Figs. 1-3 show the MSEs of the RSL-based, the SNR-based, and the SP-based path attenuation estimators for $\alpha=$ 
$0.5 \mathrm{~dB}, 3 \mathrm{~dB}$, and $18 \mathrm{~dB}$, respectively. It can be seen from the three figures that the simulated MSEs get close to the theoretical MSE approximations with the increase of $L$. For the RSL-based estimator, the approximation is poor for small $L$, as can be seen in Fig. 1. Nonetheless, the approximation is very accurate for the SNR-based estimator and the SP-based estimators, in particular for large $L$. Note that the poor performance for small $L$ is due to the error of the Tylor expansion for small $L$ is relatively large.

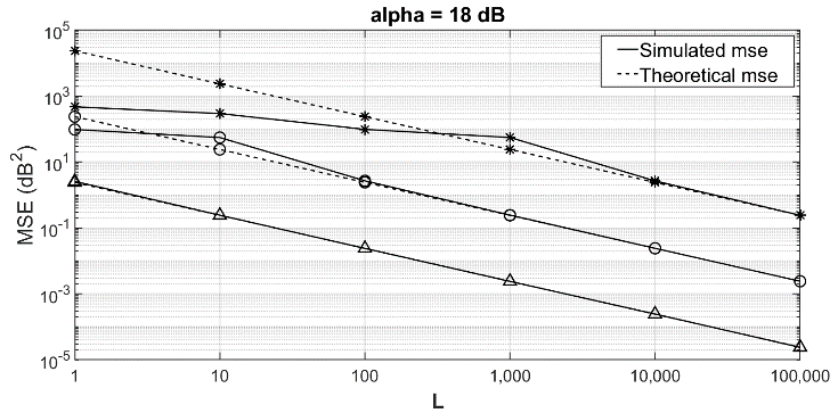

Fig. 3 MSE of the SP-based attenuation estimator at $\mathrm{SNR}=-10 \mathrm{~dB}$ $(*), 10 \mathrm{~dB}(\mathrm{o})$ and $30 \mathrm{~dB}(\Delta)$ for $\alpha=18 \mathrm{~dB}$.

Fig. 4 shows the MSEs with different SNR for the three path attenuation estimators for $L=10,000$ and 100,000, respectively. As an example, if the symbol rate of a QPSK system is $100 \mathrm{Mb} / \mathrm{s}, L=$ 100,000 corresponds to an observation window length of $2 \mathrm{~ms}$. For the RSL-based estimator, as can be seen from Fig. 4 (a), the simulated $m s e_{1}$ with larger $L$ is closer to the theoretical approximation of $m s e_{1}$, which is consistent with Fig. 1; From Fig. 4(b) and (c), it can be seen that the approximation accuracy improves with the increase of $\alpha$. When the $\alpha$ is small, the performance of the RSL-based path attenuation estimator can be better than $0.01 \mathrm{~dB}$ for high SNR or CNR (i.e., the MSE is lower than $10^{-4} \mathrm{~dB}^{2}$ ). However, its performance becomes worse as $\alpha$ increases. By comparing the three sub-figures in Fig. 4, with the increase of $\alpha$, the advantage of the SNR-based method and the SP-based method becomes more significant. The performance of the SNR-based method is very close to that of the SP-based method when the SNR is low but degrades as the SNR increases. When $\alpha=0.5 \mathrm{~dB}$ and $L=10,000$, compared with the RSL-based estimator, the SP-based path attenuation estimator shows performance advantage when the SNR is lower than $20 \mathrm{~dB}$. When $L$ increases to 100,000 symbols, the SP-based method has the best performance, for the SNR of consideration in this paper.

\section{CONCLUSION}

In this work, the path attenuation estimations based on RSL, SNR, and SP are investigated. The theoretical biases and MSEs are given by using first-order Taylor approximation. Both the theoretical and the simulated results show that the error of the RSL-based method can be lower than $0.01 \mathrm{~dB}$ for high SNR without considering quantization errors. The SNR-based method has good performance for low SNR region, but the performance becomes worse as SNR increases. The proposed SP-based method shows the best performance in most cases. The effects of the observation window lengths and the values of the path microwave attenuation are also studied in this work. Numerical results also show that a longer observation window length can improve the performance of SNRbased and SP-based attenuation estimators.

\section{ACKNOWLEDGEMENT}

This work was supported in part by Australian Research Council Discovery Projects DP190100786.

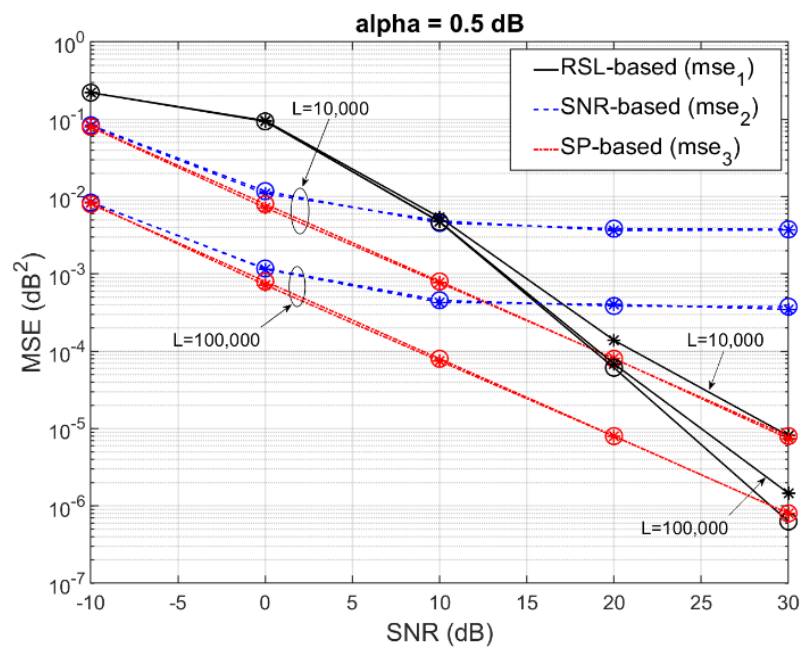

(a)

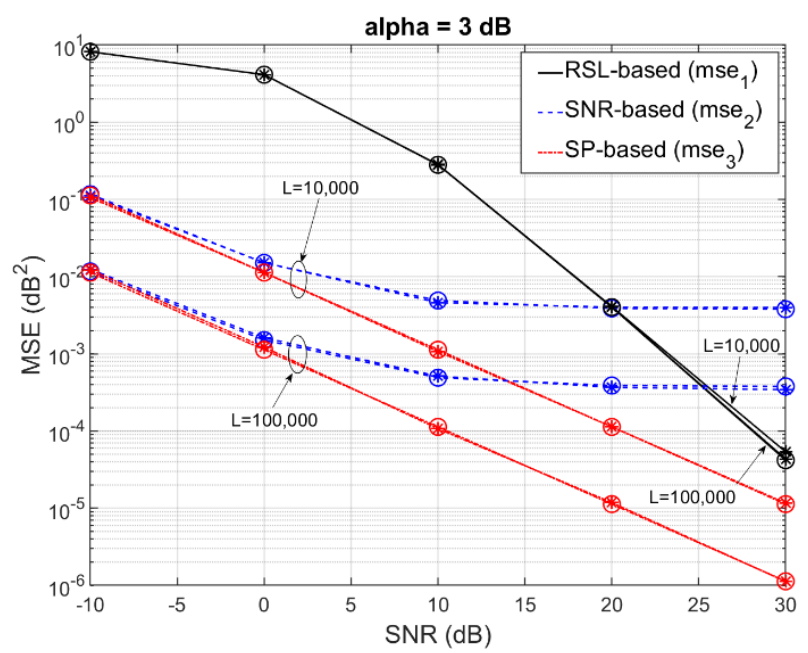

(b)

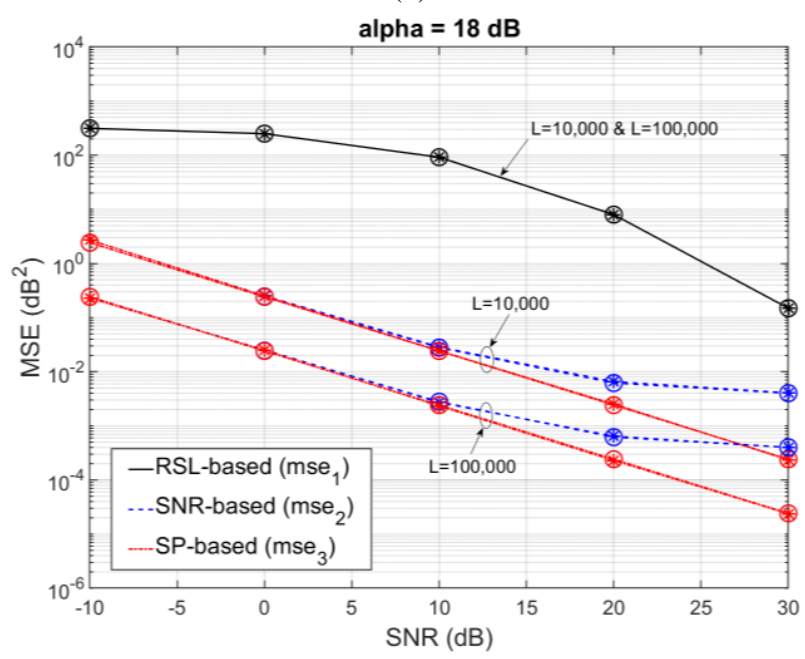

(c)

Fig. 4 The simulated MSE (*) and the theoretical MSE (o) of the three path attenuation estimators with different SNR when $\alpha=0.5$ $\mathrm{dB}, 3 \mathrm{~dB}$ and $18 \mathrm{~dB}$, respectively. 


\section{REFERENCES}

[1] H. Messer, A. Zinevich, and P. Alpert, "Environmental monitoring by wireless communication networks," Science (80-. )., vol. 312, no. 5774, p. 713, 2006.

[2] X. Shen et al., "3-D tomographic reconstruction of rain field using microwave signals from LEO satellites: principle and simulation results," IEEE Trans. Geosci. Remote Sens., vol. 57, no. 8, pp. 5434-5446, 2019.

[3] A. Dissanayake and J. Allnutt, "A prediction model that combines rain attenuation and other propagation impairments along earth-satellite paths," IEEE Trans. Antennas Propag., vol. 45, no. 10, pp. 1546-1558, 1997.

[4] Z. Elabdin, M. R. Islam, O. O. Khalifa, and H. E. A. Raouf, "Mathematical model for the prediction of microwave signal attenuation due to duststorm," Prog. Electromagn. Res. M, vol. 6, pp. 139-153, 2009.

[5] R. L. Olsen, D. V. Rogers, and D. B. Hodge, "The aRb relation in the calculation of rain attenuation," IEEE Trans. Antennas Propag., vol. 26, no. 2, pp. 318-329, 1978.

[6] A. J. Gasiewski, "Numerical sensitivity analysis of passive ehf and smmw channels to tropospheric water vapor, clouds, and precipitation," IEEE Transactions on Geoscience and Remote Sensing, vol. 30, no. 5. pp. 859870, 1992.

[7] R. Lhermitte, "A 94-GHz doppler radar for cloud observations," Journal of Atmospheric and Oceanic Technology, vol. 4, no. 1. pp. 36-48, 1987.

[8] D. Giuli, A. Toccafondi, G. B. Gentili, and A. Freni, "Tomographic reconstruction of rainfall fields through microwave attenuation measurements," Journal of Applied Meteorology, vol. 30, no. 9. pp. 1323-1340, 1991.

[9] H. Leijnse, R. Uijlenhoet, and J. N. M. Stricker, "Rainfall measurement using radio links from cellular communication networks," Water Resour. Res., vol. 43, no. 3, pp. 1-6, 2007.

[10] A. Zinevich, P. Alpert, and H. Messer, "Estimation of rainfall fields using commercial microwave communication networks of variable density," Adv. Water Resour., vol. 31, no. 11, pp. 1470-1480, 2008.

[11] H. Messer and O. Sendik, "A new approach to precipitation monitoring: acritical survey of existing technologies and challenges," IEEE Signal Process. Mag., vol. 32, no. 3, pp. $110-122,2015$.

[12] A. Gharanjik, M. R. Bhavani Shankar, F. Zimmer, and B. Ottersten, "Centralized rainfall estimation using carrier to noise of satellite communication links," IEEE J. Sel. Areas Commun., vol. 36, no. 5, pp. 1065-1073, 2018.

[13] D. R. Pauluzzi and N. C. Beaulieu, "A Comparison of SNR Estimation Techniques for the AWGN Channel," IEEE transacations Commun., vol. 48, no. 10, pp. 1681-1691, 2000.

[14] Y. Chen and N. C. Beaulieu, "Maximum likelihood estimation of SNR using digitally modulated signals," ieee transacations Wirel. Commun., vol. 6, no. 1, pp. 210-219, 2007.

[15] J. G. Proakis and M. Salehi, Digital communications. New York: McGraw-hill, 2001.

[16] H. Messer, A. Zinevich, and P. Alpert, "Environmental sensor networks using existing wireless communication systems for rainfall and wind velocity measurments," IEEE Instrum. Meas. Mag., vol. 15, no. 2, pp. 32-38, 2012.

[17] H. Leijnse, R. Uijlenhoet, and J. N. M. Stricker, "Rainfall measurement using radio links from cellular communication networks," Water Resour. Res., vol. 43, no. 3, pp. 1-6, 2007.

[18] R. M. Gagliardi, C. M. Thomas, and C. M. Thomas, "PCM data reliability monitoring through estimation of signal-tonoise ratio," IEEE Trans. Commun. Technol., vol. 16, no. 3, pp. 479-486, 1968.

[19] N. S. Alagha, "Cramer-Rao bounds of SNR estimates for BPSK and QPSK modulated signals," IEEE Commun. Lett., vol. 5, no. 1, pp. 10-12, 2001.

[20] D. Giuli, L. Facheris, and S. Tanelli, "Microwave tomographic inversion technique based on stochastic approach for rainfall fields monitoring," IEEE Trans. Geosci. Remote Sens., vol. 37, no. 5 II, pp. 2556-2568, 1999. 